\title{
Evaluation of a dental nurse-led triage system in a private dental practice during the COVID-19 pandemic
}

\author{
Angela Cowell, ${ }^{1}$ Louise Goodwin, ${ }^{1}$ Katherine Hare*1 and Colin Campbell ${ }^{1}$
}

\section{Key points}

Demonstrates the effectiveness of a dental nurse-led triage model during the cessation of face-to-face care in the COVID-19 pandemic.
Outlines the presenting problems of patients and their uptake of treatment upon practice reopening.
Suggests that this model is applicable to a routine out-of-hours service as well as at times of practice closures, and that other practices may wish to adopt it

\begin{abstract}
Objectives To evaluate a dental nurse-led triage system at a private dental practice in England after the cessation of face-to-face care during the COVID-19 pandemic; to assess types of presenting problems and their management upon practice reopening at alert level 4; and to explain the benefits of dental nurse-led triage and its relevance to other practices.
\end{abstract}

Methods This retrospective service evaluation used anonymised data gathered for the purpose of assessing and triaging patients when they could not be seen face-to-face. Effectiveness of the triage system was assessed using predetermined criteria.

Results Seventy patients were triaged during a 12-week period; $68.5 \%$ of patients were managed by the triaging dental nurse without escalation to a dentist and $77 \%$ of patients called only once. The most common presenting complaint was pain, followed by loose crown/bridge and fractured crown/tooth/bridge. Sixty-one percent of all patients accepted a face-to-face appointment upon reopening of the dental practice.

Conclusions This study demonstrates the effectiveness of a dental nurse-led triage model where dental nurses have the skills and experience required to manage patients at first contact. This model would be applicable to other practices in the case of future emergency closures, as well as a routine out-of-hours service.

\section{Introduction}

On 23 March 2020, the UK government instructed the population to stay at home and only leave their home for limited purposes in order to halt the spread of coronavirus. ${ }^{1}$ This was followed on 25 March by a letter (issue 3) from NHS England and NHS improvement, stating that 'all routine non-urgent general dental care should be stopped and deferred with all practices establishing a remote urgent care service providing telephone triage for their patients with urgent needs during normal working hours.'2

${ }^{1}$ The Campbell Clinic (Private Dental Practice), Nottingham, UK.

*Correspondence to: Katherine Hare

Email address: kath@campbell-clinic.co.uk

Refereed Paper

Accepted 8 September 2020

https://doi.org/10.1038/s41415-020-2177-3
An unprecedented situation suddenly emerged whereby dental practices essentially closed their doors to patients, with the only care offered being the ' $3 \mathrm{As}$ ' - telephone advice, analgesia and, where appropriate, antimicrobials. ${ }^{3,4,5}$

NHS practices were expected to undertake this during their normal working hours as part of their existing contract. ${ }^{6}$ Where necessary, patients could be referred to urgent dental centre hubs for treatment.

This article focuses on a single private practice in England which provides a full range of general and specialist dental treatments. For many years, the clinic has run an effective dental nurse-led triage emergency service seven days a week for their patients. The existing skills in this field allowed the practice to quickly move away from face-to-face care from 23 March 2020, and therefore, the authors wish to share this model and report their findings of how emergency patients were triaged and managed. This system enabled the practice to restart efficiently to see emergency patients when dental practices in England were advised to reopen on 8 June $2020 .^{8}$

Dental nurse-led triage has been considered as a model for out-of-hours emergency services for a long time; ${ }^{9,10}$ however, there is very little evidence published. In general medical practice, nurse triage has been demonstrated to reduce the rate of overall GP contacts by $16 \%$ and therefore redistribute the primary care workload. ${ }^{11}$ The authors share this model and define measures of its effectiveness, with the aim that it could be simply and confidently put into place by other dental teams in the future, in the case of future local lockdowns due to COVID-19 (as has already been required in Leicester) ${ }^{12}$ or other emergency closures, as well as a routine out-of-hours service.

The model described here demonstrates how the dental team can work together effectively, even while being geographically remote and separate from a dental practice. 


\section{Method}

\section{Patient cohort}

Anonymised data were retrospectively acquired from a triage system set up specifically for the period during which dental practices were closed due to the COVID-19 pandemic. All identifiers were removed for analysis; therefore, patient consent was not sought and no patients were excluded from this study. HRA (Health Research Authority) decision tools $^{13,14}$ (an authoritative decision in line with UK GAfREC) were used to confirm this service evaluation did not require ethical approval.

\section{Setting}

All data relate to patients at a single private dental practice in England. Triage system was by phone consultation.

\section{Context}

In March 2020, the UK went into lockdown as a result of the COVID-19 pandemic. This triage system went live the day after this dental practice closed to all patients on Monday, 23 March 2020. It continued to triage and offer advice by phone until the end of the first week of reopening - Friday, 12 June 2020. From 8-19 June 2020, all triaged patients who accepted an appointment were assessed in the practice, during which time the UK remained at alert level $4 .^{15}$

\section{Triage system}

Calls to the practice were initially taken by reception staff (working from home) during normal working hours through a diverted

Table 1 Patient demographics

\begin{tabular}{l|l|l|l}
\hline Age range & Male & Female & Total \\
\hline $21-30$ & 2 & 4 & 6 \\
\hline $31-40$ & 1 & 5 & 6 \\
\hline $41-50$ & 6 & 7 & 13 \\
\hline $51-60$ & 3 & 12 & 15 \\
\hline $61-70$ & 8 & 6 & 14 \\
\hline $71-80$ & 5 & 8 & 13 \\
\hline $80+$ & 2 & 1 & 3 \\
\hline Total & 27 & 43 & 70 \\
\hline
\end{tabular}

phone number. Patients experiencing problems were then transferred to the triage dental nurse. Out-of-hours calls were taken as normal through the practice's emergency mobile phone number. Current orthodontic patients were managed directly by the practice's specialist orthodontist.

The triage dental nurse (who was the same nurse throughout) was able to access the patient's clinical records through Dentally, which is a cloud-based system, and discuss their dental problem and concerns in order to complete a triage pro forma for each patient. Appendix 1 shows the triage pro forma detailing patient demographics, date of call, the date of the patient's last clinic appointment, nature of dental problem, action required and advice given. Where possible, the patient complaint was recorded using the terms they used, and for all patients, clear instructions were given to contact the service again should there be deterioration or no improvement. This is described as 'clear worsening advice given' on the triage form (Appendix 1).

Where needed, calls were escalated by the triage dental nurse to the dentist on call ('second contact') who would telephone the patient directly. For complex issues, the patient was escalated further to the clinical lead dentist ('third contact'). Both dentists had access to patient clinical records. Patients requiring ongoing monitoring and contact were followed up periodically.

As new cases of COVID-19 declined and dental services in England could resume, ${ }^{8}$ the patients listed on the triage spreadsheet were discussed at the weekly online multidisciplinary team meeting and assigned to an appropriate clinician. This enabled reception staff to arrange for all triaged patients to be offered an appointment during alert level $4{ }^{15}$ with low-risk aerosol generating procedures (AGPs). ${ }^{16}$

Table 2 The reason for call was recorded and analysed by action taken at the time, by nurse contact only or escalation to dentist

\begin{tabular}{|c|c|c|c|c|c|}
\hline Primary reason for call & Advice (nurse) & $\begin{array}{l}\text { Advice and ongoing } \\
\text { contact (nurse) }\end{array}$ & Passed to dentist & $\begin{array}{l}\text { Passed to clinical lead } \\
\text { dentist }\end{array}$ & Total number \\
\hline Fractured tooth/crown/bridge & 8 & 0 & 3 & 0 & 11 \\
\hline Gingival problem & 3 & 0 & 1 & 0 & 4 \\
\hline Implant problem & 2 & 0 & 3 & 0 & 5 \\
\hline Loose crown/bridge & 11 & 0 & 3 & 0 & 14 \\
\hline Lost filling & 3 & 0 & 2 & 0 & 5 \\
\hline Orthodontic problem & 1 & 0 & 0 & 0 & 1 \\
\hline Pain & 12 & 2 & 4 & 3 & 21 \\
\hline Reassurance & 1 & 0 & 0 & 0 & 1 \\
\hline Swelling & 0 & 0 & 2 & 0 & 2 \\
\hline Other & 5 & 0 & 0 & 1 & 6 \\
\hline Total & 46 & 2 & 18 & 4 & 70 \\
\hline
\end{tabular}




\section{Monitoring effectiveness of the triage} system

A number of criteria were proposed to monitor the effectiveness of this triage system. These criteria were: minimising the need to call multiple times; patient concerns managed by triage dental nurse; appropriate escalation of patient to dentist; and escalation of patient on first contact for severe problems.

\section{Results}

\section{Primary evaluation}

Seventy patients (27 male, 43 female) were added to the triage list as they required appointments for treatment upon the reopening of the practice. The mean age was 57 years old (age range 21-89) (see Table 1).

The most common primary reason for patients contacting the practice was pain $(\mathrm{n}=21)$, followed by a loose crown/bridge ( $\mathrm{n}=14)$ or fractured tooth/crown/bridge $(\mathrm{n}=11)$. Of the 70 patients triaged, 48 (68.5\%) were managed by advice and ongoing nurse contact without needing input from a dentist. Only 18 (25\%) patients were passed to the second contact (dentist) and four (5.7\%) required escalation to the third contact (clinical lead dentist) (see Table 2). The most common presenting complaint of patients who required escalation to the second and third contacts was pain.

Antimicrobials were prescribed by a dentist according to FGDP standards ${ }^{5}$ for only 5/70 triaged patients and no patients required onwards referral to urgent dental centres.

Patients had to wait between 8-84 days between their first phone call to the triage service and their clinical appointment. All patients understood the practice was closed when phone advice was given and were told they would be seen once the practice was allowed to reopen.

Twenty-seven of the 70 triaged patients (38.5\%) declined an appointment when offered one. The reasons for this are in Table 3.

Table 3 As the practice prepared to reopen, all patients were contacted to be offered an appointment; however, not all accepted the appointment offered, for reasons detailed here

\begin{tabular}{l|l}
\hline Reason for declining appointment & Number of patients \\
\hline Has future appointment booked and wants to wait & 7 \\
\hline Problem has settled & 6 \\
\hline Patient/relative shielding & 3 \\
\hline Seeing own GDP & 4 \\
\hline Other (including no response) & 7 \\
\hline Total & $\mathbf{2 7}$ \\
\hline
\end{tabular}

Sixteen patients called multiple times; however, ten of these were never escalated to a dentist and always dealt with by the triage nurse. Only one of the multiple callers required contact from the clinical lead dentist. Fourteen $(87.5 \%)$ of these 16 frequent callers accepted appointments when offered.

\section{Secondary evaluation}

Of the 43 patients seen for appointments, 13 patients had problems relating to ongoing treatment whereas 30 patients had a new problem. Fifteen patients were assessed at their first clinical appointment, ten were able to have a 'temporary fix' and 18 had a 'permanent fix' (see Table 4).

\section{Discussion}

This service evaluation during the COVID-19 pandemic offers a practice-based perspective from those already published in a hospitalbased acute care service ${ }^{17}$ and an urgent dental care centre. ${ }^{7}$ The practice was in an agile position to respond to this unprecedented situation to move away from face-to-face dental care and remote working because of the IT and communications systems already in place, such as the ability to divert phone lines and a cloud-based clinical dental records system. Other practices may have been in a different situation, as this practice received calls from patients who could not contact their usual
Table 4 Upon initial assessments (at alert level 4), patients' problems were either assessed, fixed or temporarily fixed as detailed here. Some problems related to ongoing treatment, while others were a new issue

\begin{tabular}{l|l|l|l}
\multirow{2}{*}{ Action } & \multicolumn{2}{|c|}{ Problem relating to: } & \multirow{2}{*}{ Total } \\
\cline { 2 - 4 } & Ongoing treatment & New issue & 15 \\
\hline Assess & 2 & 13 & 10 \\
\hline Temporary fix & 6 & 4 & 18 \\
\hline Fix & 5 & 13 & $\mathbf{4 3}$ \\
\hline Total & $\mathbf{1 3}$ & $\mathbf{3 0}$ & \\
\hline
\end{tabular}

dentist. The weekly online multidisciplinary team meeting of clinicians allowed discussions regarding patient management and, due to the data recorded, clinicians could grade patients according to priority need with ease.

This triage service built upon the foundations of an existing nurse-led, on-call, out-of-hours triage system which has been in place for over six years at the practice. An audit of this system throughout 2019 showed that 31 calls were received by the triage nurse, of which 16 were escalated to the on-call dentist. During the 12-week period of nurse-led triage due to COVID-19, 70 calls were received, of which 48 (68.5\%) were managed solely by the triage nurse and 22 (45.7\%) required onwards referral to a dentist. It is expected that a higher number of calls would be received during this period because it includes all patients contacting the practice with problems, rather than an out-ofhours service as no face-to-face appointments could be offered.

The effectiveness of this triage model was defined as: minimising the need to call multiple times; patient concerns managed by triaging dental nurse; appropriate escalation of patient to dentist; and escalation of patient on first contact for severe problems.

In this study, $68.5 \%$ of calls were managed by the triage dental nurse. This shows that this is well within their skillset and is an effective division of workload for the dental team. Only 16 of the total 70 patients called multiple times, and ten of these were never escalated and always dealt with by the triage dental nurse. This again demonstrates the ability of the dental nurse to manage and communicate with patients, as further clinical input was not required. While escalation to a dentist happened on 22 occasions in total, only five of these patients were escalated on their second or third call (rather than their first call), suggesting 65/70 (93\%) of patients were appropriately managed at their first contact. At no point did a dentist 
suggest that action taken at the time of the call was inappropriate during the triage period or having subsequently seen the patient. All contacts which were escalated to a dentist were deemed appropriate referrals. In the absence of any published evidence, these results were felt to broadly meet 'effectiveness' criteria and provide a means by which to measure effectiveness of future dental nurse-led triage.

As a private practice with specialisms, a different mix of problems during the COVID19 pandemic may have been seen compared to those previously reported for out-of-hours emergency services in general ${ }^{18}$ or regional ${ }^{10}$ facilities. Both of these had approximately half of their patients complaining of pain. In this evaluation, while the most common primary reason for patients contacting the practice was pain ( $n=21,30 \%$ ), this is different to the practice's routine audits of emergency and unplanned treatment before COVID-19, where patients most frequently present with loose/ lost bridge followed by a loose/lost crown and then pain. It seems that pain was more prevalent during this time, and although this cannot be definitively answered, the authors speculate it is due to patients seeking advice as they were aware of the service limitations and the uncertainty as to when a face-to-face appointment would be available in the future.

The triage sheet (Appendix 1) explains that patients should be recommended paracetamol. This was current advice at the time the triage system was initiated (March 2020), as there were concerns over ibuprofen and susceptibility to contracting COVID-19; however, these were later found to have insufficient evidence. ${ }^{19}$ It may be that this had an effect on patients' pain control. It is interesting to note that often patients had not taken pain relief before speaking to the triage nurse. This is an issue that is also commonly encountered in the out-of-hours emergency service and patients require advice regarding this.

Grossman et al. ${ }^{17}$ highlight the importance of managing conditions such as mild to moderate orofacial pain during the pandemic as urgent, rather than routine, due to the restrictions in accessing services. Interestingly, when the patients who had complained of pain could be offered an appointment in practice, approximately half of them declined this. Indeed, one unexpected result was that 27/70 triaged patients $(38.5 \%)$ declined a face-toface urgent appointment when the practice reopened. This was a higher proportion than the authors expected, with a considerable proportion of these patients happy to wait longer to be seen because they already had a scheduled appointment with this practice or their own GDP. For several others, the problem had settled and patients felt they did not merit an appointment. While three patients declined appointments because they or a relative were shielding, ${ }^{20}$ no patients declined appointments due to specific safety concerns in attending a dental practice.

When the practice reopened, the authors anticipated that patients would mainly be seen regarding problems they experienced due to ongoing treatment being disrupted by the loss of face-to-face care; however, this was not the case. The pause in ongoing treatment did not appear to result in problems for many patients, including all those who were mid-treatment and who did not need to contact the triage service. A large proportion of patients could only be assessed or have a temporary fix when first seen, as they required a high-risk AGP and the practice followed a stepwise approach as advised $^{8,16}$ in undertaking low-risk AGPs first. With the change to alert level 3 on 19 June, these patients were subsequently managed with the full complement of treatment they required to resolve their problems.

This study has some limitations as data collection was not fully defined before the cessation of face-to-face treatment and subsequent implementation of this triage model. With very little notice of practice closures due to the escalation of the COVID19 pandemic, it would have been difficult to set this up as a prospective study, hence this evaluation can only be retrospective. However, it is important to note that the NHS e-triage data collection only began on 4 June 2020,21 hence this study covers a period before this for which there is no NHS data.

In addition, no data were collected regarding patient satisfaction with the triage process. Patient satisfaction could be construed to be largely positive, due to the low level of repeat callers and subsequent escalation to dentists, as it is reasonable to assume any patient unsatisfied with the handling and resolution of their problem would call again. According to the triaging dental nurse, the majority of patients were grateful to be given advice on managing their problem and welcomed reassurance they would be seen as soon as possible once this was allowed, although this is anecdotal. Future studies of dental nurse-led triage would benefit from the inclusion of a measure of patient satisfaction.

\section{Conclusions}

This study demonstrates the effectiveness of a dental nurse-led triage system, as the majority of patients were effectively managed by a single phone call to the triage dental nurse. It shows effective teamwork as dental nurses have the skills and experience required to manage patients at first contact. This built on the foundations of an existing on-call nurse-led triage system which allowed dentists to be contacted when their input was needed. In this case, during the COVID19 pandemic, it allowed the dental team to prepare for reopening the practice while having a robust support system (second and third dentist contact) in place for escalation of patient calls where necessary. It may be necessary to activate this triage model once more as it is likely that local lockdowns will be needed until there is a nationally low and controlled $\mathrm{R}$ number, or a vaccine is found and implemented. Although this is a smallscale study in a single dental practice, it has many commonalities to other dental practices, regions and nations throughout the COVID19 pandemic, and many others may benefit from its implementation.

\section{Acknowledgements}

The authors are grateful to Nish Yadev and Sharon Charnley for their help in managing patients.

\section{Conflict of interest}

The authors have no conflicts of interest to declare.

\section{References}

1. UK Government. Prime Minister's statement on coronavirus (COVID-19): 23 March 2020. 2020. Available at https://www.gov.uk/government/speeches/ pm-address-to-the-nation-on-coronavirus-23march-2020 (accessed August 2020).

2. NHS England. Preparedness Letter for Primary Dental Care 25th March. 2020. Available at https://www. england.nhs.uk/coronavirus/wp-content/uploads/ sites/52/2020/03/issue-3-preparedness-letter-forprimary-dental-care-25-march-2020.pdf (accessed August 2020).

3. SDCEP. Management of Acute Dental Problems During COVID-19 Pandemic. 2020. Available at https://www. sdcep.org.uk/wp-content/uploads/2020/03/SDCEPMADP-COVID-19-guide-300320.pdf (accessed August 2020)

4. SDCEP. Drugs for the Management of Dental Problems During COVID-19 Pandemic. 2020. Available at https:// www.sdcep.org.uk/wp-content/uploads/2020/05/ SDCEP-MADP-COVID-19-drug-supplementupdate-110520.pdf (accessed August 2020).

5. FGDP. Antimicrobial prescribing standards (2nd edition) 2014. Available online at https://www.fgdp.org.uk/ antimicrobial-prescribing-standards/contents (accessed August 2020).

6. NHS England. Letters, updates and additional guidance for dental teams. 2020. Available at https://www. england.nhs.uk/coronavirus/publication/preparednessletters-for-dental-care/ (accessed August 2020). 
7. Carter E, Currie C C, Asuni A et al. The first six weekssetting up a UK urgent dental care centre during the COVID-19 pandemic. Br Dent J 2020; 228: 842-848.

8. NHS England. Resumption of dental services in England. 2020. Available at https://www.england.nhs.uk/ coronavirus/wp-content/uploads/sites/52/2020/03/ Urgent-dental-care-letter-28-May.pdf (accessed August 2020).

9. Evans D J, Smith M P, Grant S M B, Crawford M A, Bond J. Outofhours Emergency Dental ServicesDevelopment of One Possible Local Solution. Br Dent J 2001; 191: 550-554.

10. Topping G V A. Outofhours Emergency Dental ServicesEvaluation of the First Year of a Pilot Project in Fife. Br Dent J 2005; 198: 193-197.

11. Campbell J L, Fletcher E, Britten $\mathrm{N}$ et al. The Clinical Effectiveness and Cost-Effectiveness of Telephone Triage for Managing Same-Day Consultation Requests in General Practice: A Cluster Randomised Controlled Trial Comparing General Practitioner-Led and NurseLed Management Systems With Usual Care (The ESTEEM Trial). Health Technol Assess 2015; 19: 1-212.

12. UK Government. Plans for managing the coronavirus (COVID-19) outbreak in Leicester. 2020. Available at https://www.gov.uk/government/speeches/ local-action-to-tackle-coronavirus (accessed August 2020).

13. NHS Health Research Authority. Do I need NHS REC review? Available online at http://www.hradecisiontools.org.uk/ethics/ (accessed August 2020).

14. NHS Health Research Authority. Is my study research? Available online at http://www.hra-decisiontools.org. uk/research/ (accessed August 2020).

15. UK Government. COVID Alert Levels. 2020. Available at https://assets.publishing.service.gov.uk/government/ uploads/system/uploads/attachment_data/ file/884352/slides_-_11_05_2020.pdf (accessed August 2020).

16. College of General Dentistry and FGDP. Implications of COVID-19 for the safe management of general dental practice: A practical guide. 2020. Available at https://www.fgdp.org.uk/sites/fgdp.org.uk/files/ editors/FGDP\%20CGDent\%20Implications\%20of\%20 COVID-19\%20for\%20the $\% 20$ safe $\% 20$ management $\% 20$ of $\% 20$ general $\% 20$ dental $\% 20$ practice $\% 2016 \% 20$ June $\% 202020 \% 20$ ed1.1.pdf (accessed August 2020).

17. Grossman S, Sandhu P, Sproat C, Patel V. Provision of dental services at a single institution in the UK's epicentre during the COVID-19 pandemic. Br Dent J 2020; 228: 964-970.
18. Portman-Lewis S. An Analysis of the OutOfHours Demand and Treatment Provided by a General Dental Practice Rota Over a Five-Year Period. Prim Dent Care 2007; 14: 98-104.

19. UK Government. Commission on Human Medicines advice on ibuprofen and coronavirus (COVID-19). 2020. Available at https://www.gov.uk/government/news/ commission-on-human-medicines-advice-on-ibuprofenand-coronavirus-covid-19 (accessed August 2020).

20. Public Health England. Guidance on shielding and protecting people who are clinically extremely vulnerable from COVID-19. 2020. Available at https://www.gov.uk/government/publications/ guidance-on-shielding-and-protectingextremely-vulnerable-persons-from-covid-19/ guidance-on-shielding-and-protecting-extremelyvulnerable-persons-from-covid-19 (accessed August 2020).

21. British Dental Association. COVID-19 guidance and standard operating procedure: For the provision of urgent dental care in primary care dental settings (from 8 June 2020) and designated urgent dental care provider sites. 2020. Available at https://bda.org/ advice/Coronavirus/Documents/covid-19-urgentdental-care-sop.pdf (accessed August 2020).

\section{Appendix 1 The triage pro forma used by the dental nurse to collect information from the patient and record action taken. Paracetamol advice was current at initiation of triage system (23 March 2020)}

\section{COVID-19 dental triage}

Patient name:

Patient date of birth:

First contact (dental nurse): Date of call:

Second contact (dentist): Last appointment at The Campbell Clinic was:

Third contact (lead dentist):

\section{Reason for call}

Pain

Loose crown/bridge

Post-operative bleeding

Lost filling

Fractured tooth/crown/bridge

Swelling $\square \quad$ Denture problem (broken, lost, painful)

$\square$ Reassurance

$\square$ Implant problem

$\square$ Orthodontic breakage

$\square$ Gum problem

$\square$ Other (document below)

Does the patient have any other symptoms not listed here? $\quad$ Yes $\square \quad$ No

Details of call:

\section{Action to be taken - please tick:}

COVID-19 reassurance - treatment coordinator to contact and monitor until further notice

COVID-19 call closed with self-help and clear worsening instructions

COVID-19 call passed to second contact

COVID-19 call passed to third contact

COVID-19 urgent treatment required - arrangements made to see patient

\section{Advice given:}

Clear worsening instructions given 